\title{
Cerebro: A Wearable Solution to Detect and Track User Preferences using Brainwaves
}

\author{
Mohit Agarwal \\ Georgia Institute of Technology \\ magarwal37@gatech.edu
}

\author{
Raghupathy Sivakumar \\ Georgia Institute of Technology \\ siva@gatech.edu
}

\begin{abstract}
In this work, we consider the problem of detection and interpretation of user preferences using their brainwaves. The specific goal in this context is to determine the preference ranking for a set of objects by solely relying on the brain activity of a user who is wearing an EEG headset wearable. We first establish the feasibility of object ranking (based on an EEG wearable) by a trial and error based analysis of the EEG signals. We then present a machine learning algorithm Cerebro, which can learn the specific nuances of the user's brainwaves for preferences to accurately rank the objects. We measure the accuracy of the algorithm in terms of the Normalized Discounted Cumulative Gain (NDCG), and show that it performs well when trained on 7 objects, and evaluated on 3 objects for the 14 users.
\end{abstract}

\section{ACM Reference Format:}

Mohit Agarwal and Raghupathy Sivakumar. 2019. Cerebro: A Wearable Solution to Detect and Track User Preferences using Brainwaves. In The 5th ACM Workshop on Wearable Systems and Applications (WearSys'19), fune 21, 2019, Seoul, Republic of Korea. ACM, New York, NY, USA, 6 pages. https://doi.org/10.1145/3325424.3329660

\section{INTRODUCTION}

Knowledge of a user's preferences can be quite useful in several different contexts. For example, Amazon, the online retailer, sells over 600 million products. The Amazon landing page, on the other hand, can reasonably present only 50-60 different products on a computer, and fewer on a mobile device. When a user arrives at the landing page, Amazon would ideally like to present those products that are of relevance to the user. Knowing the user's preferences at that point in time can help Amazon do so effectively.

Sophisticated user personalization models are routinely employed today by a retailer such as Amazon based on cues such as past purchases, searches, and items saved in cart. There are

Permission to make digital or hard copies of all or part of this work for personal or classroom use is granted without fee provided that copies are not made or distributed for profit or commercial advantage and that copies bear this notice and the full citation on the first page. Copyrights for components of this work owned by others than ACM must be honored. Abstracting with credit is permitted. To copy otherwise, or republish, to post on servers or to redistribute to lists, requires prior specific permission and/or a fee. Request permissions from permissions@acm.org.

WearSys'19, June 21, 2019, Seoul, Republic of Korea

(C) 2019 Association for Computing Machinery.

ACM ISBN 978-1-4503-6775-2/19/06 ..\$15.00

https://doi.org/10.1145/3325424.3329660 other contexts as well beyond online commerce where the ability to understand user preferences has significance.

Meanwhile, over the last couple of decades, rapid strides have been made in the domain of sensing and interpreting brain activity using electroencephalogram (EEG). Unlike its more involved counterparts such as magnetic resonance imaging (MRI) and functionalMRI, one of the distinct advantages of EEG is that the sensors can be used in a non-obtrusive user-friendly fashion. Figure 1 shows an off-the-shelf EEG "headset" wearable (EHW) that looks no different from audio headphones. This advantage makes EEG a prime candidate for mainstream applications that reliably rely on brainwaves for understanding user thoughts. Advances in the understanding of brain architecture and functioning, coupled with sophisticated signal processing techniques, has allowed for EEG based detection of user actions (e.g. blinks) and thoughts (e.g. motor imagery and error response).

In this work, we consider the intersection of the aforementioned domains. Specifically, we consider the detection and interpretation of user preferences using only the brain waves of the user detected using an off-the-shelf EEG wearable.

We consider this problem in the specific context of ranking a given set of objects based on a user's preferences. Thus, given a set of objects $O S=\left\{o_{1}, o_{2}, \ldots, o_{N}\right\}$, we consider the problem of determining the respective ranks of the objects $R S=\left\{r_{1}, r_{2}, \ldots, r_{N}\right\}$, where $1<=r_{i}<=N$, by only relying on the brain activity of a user who is wearing an EEG headset wearable. The following is a summary of our key contributions:

- Using an EEG dataset obtained from 14 users observing 10 different objects (products), we first establish the feasibility of object ranking based on an EEG wearable. We do so by relying on a brute-force trial and error based analysis of the EEG signals and comparing it to the ground truth of how the users explicitly ranked the corresponding objects.

- We then present a machine learning algorithm, Cerebro, that given a training set of EEG waveforms along with rankings from a specific user, can learn the specific nuances of the user's waveforms for preferences, and when provided with only the waveforms for a new set of objects can rank those objects accurately. The key novelty of Cerebro lies in the combined use of multiple aspects of the EEG signals (N200 mean, N200 minima, and Event-related Spectral Power (ERSP)) to rank objects according to user preferences, and a mechanism to self-determine when the algorithm's ranking are accurate enough to be actionable.

- We evaluate the Cerebro solution by training the algorithm with 7 objects for the 14 users, and evaluating the accuracy with which it ranks the remaining 3 objects as compared to the user-specified rankings. 
The rest of the paper is organized as follows. In Section 2, we present some background on user preferences and EEG and define our problem formally. In Section 3, we establish the feasibility of object ranking based on EEG signals by using the dataset. In Section 4, we present the Cerebro algorithm and evaluate its accuracy performance. Finally, we present our conclusions.

\section{BACKGROUND AND PROBLEM DEFINITION}

\subsection{User Preferences}

A user's preferences influence everything from mundane purchases to social behavior to moral decisions. The neurobiology of preferences is still an emerging area of study, but it is understood that preferences are influenced by both genetics and the environment. Since preferences heavily determine a user's actions, having visibility into the preferences can help in several different scenarios. While we delve into some example scenarios later, we now briefly discuss some approaches to determine a user's preferences.

An obvious approach to learn a user's preferences is to ask the user for explicit input. For example, presenting a set of options to a user and having the user vote or rank on the options explicitly. An advantage of this approach is that the user's stated preferences are directly known. However, there are a few drawbacks: first, when user's share preferences they might not be entirely truthful and represent accurately their real preferences - this is observed routinely in pre-election polls; and second, since this approach requires explicit user involvement, it cannot be used frequently and for a large number of options.

An alternative to the explicit approach is to implicitly observe user actions and infer the user's preferences based on those actions. This is the preferred approach especially for environments such as e-commerce platforms where observing a user's actions is significantly easier than explicitly interacting with the user. A platform like Amazon observes a user's actions such as searches, clicks, time spent on a product page, additions to cart, and actual purchases to form a composite view of user's preferences and use this to appropriately optimize the options presented to the user. Video platforms such as Netflix and YouTube also rely on similar techniques to understand user preferences in order to present suggestions for users to watch next. YouTube's recommendation engine has a remarkably high success rate - over $70 \%$ of a user's watch behavior is directly from the recommended videos presented to the user ${ }^{1}$

There are some specific scenarios where it is neither possible for users to explicitly indicate preferences, nor is it possible to reliably track user actions to make meaningful inferences. For example, consider the problem of learning the preferences of a user with disabilities that preclude both explicit communication and any pertinent actions that would allow for meaningful inferences. Similarly, learning about the true preferences of young kids is a challenge.

In this work, we focus on implicit observations, but not on the user's actions that can be somewhat infrequent, but on the user's thoughts. Thoughts as a unit of observation are far more frequent,

\footnotetext{
${ }^{1}$ https://www.cnet.com/news/youtube-ces-2018-neal-mohan/
}

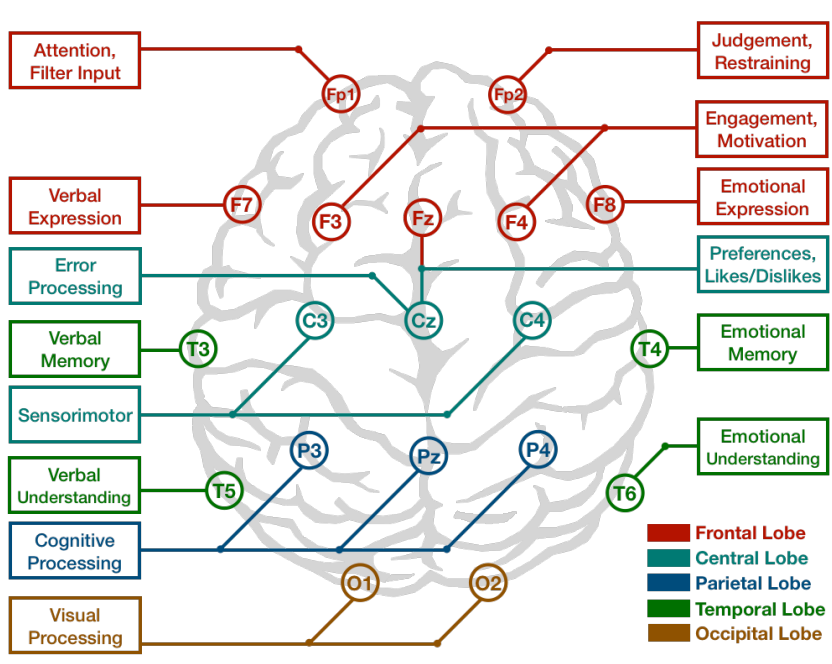

Figure 2: EEG electrodes and associated neural activity

and more seamlessly accessible, than actions. Hence, there is considerable merit in considering the observation of thoughts using EEG in order to infer user preferences.

\subsection{A Quick Primer on EEG}

EEG (Electroencephalography) is the recording of electrical activity of the brain on the scalp. This electrical activity is the derivative of synchronized electrical firings of billions of neurons inside the brain responsible for the processing and communication of a massive amount of information. The raw analog electric potentials are tapped by placing electrodes (conductive disks, often mounted in a fabric cap) over the human scalp. The raw signals are further digitized and amplified through appropriate sensing hardware. These EEG signals are a very crude representation of the brain activity, as it can only capture the macroscopic firings of the large group of neurons. With significant advances in consumer-grade EEG headsets, these signals can be reliably tapped into by the user wearing an electrode cap and digitized further. The measurement and processing of such potentials provide a window into a myriad of activities inside the brain including emotions, perception, attention, engagement, etc. [1-3]. Specific sections of the brain are responsible for different cognitive and biological functions, and the neural activity associated with those functions is recorded by placing electrodes directly above the specific regions. Fig. 2 shows the main spatial locations and the associated neural activity. For e.g., neural activity related to motor and sensory tasks is prevalent in $\mathrm{C} 3, \mathrm{C} 4, \mathrm{Cz}$ electrodes (according to 10-20 electrode system) in 8-12 Hz band. A user preference related neural component is captured over $\mathrm{Fz}$ and $\mathrm{Cz}$, in the time interval of $200-300 \mathrm{~ms}$.

Such attributes (e.g. attention, emotions, like, dislike, etc.), reflecting the user preferences associated with neurophysiological changes, and the methodologies to track through EEG, fMRI, MEG devices, are being heavily used in the advertisement industry (e.g. Coca-Cola, Campbell, GE) and is an active area of academic research [4]. EEG provides excellent time resolution (although poor spatial resolution), allowing to detect brain activity in milliseconds, 
making it an inexpensive viable candidate for understanding user preferences.

\subsection{Target Scenario and Problem Statement}

We consider a setup where a user is wearing an EEG headset while browsing through the e-commerce platform on her computer or phone. The electrode sensors continuously read raw brain signals, and the hardware platform transforms them into digital signals. The digitized brainwaves are transferred to the cloud over a wireless link for computational processing. The raw EEG signals are pre-processed (to increase the signal-to-noise ratio) and are dissected into fundamental frequency components (primarily theta and beta waves) in the cloud to search for specific patterns. The processed features are then subjected to learning algorithms to interpret their meaning. Thus, with such analysis, conscious or subconscious user preference toward the browsed or recommended item can be inferred. If multiple objects are shown at the same time, attribution methodologies are required to tie user preference to a specific item. The user-specific model in the cloud is updated based on the learned preferences of the known item, which delivers the updated personalized recommendation to the user device.

Our goal in this paper is to determine the preference ranking for a set of objects by only relying on the brain activity of a user who is wearing an EEG headset wearable. We define the mathematical formulation of the problem as follows,

Problem Definition: Consider a user $U$ presented with a set $O S$ of $N$ objects, $O S=\left\{o_{1}, o_{2}, \ldots, o_{N}\right\} . S=\left\{s_{1}, s_{2}, \ldots, s_{N}\right\}$, represents the corresponding recorded neural measures while a user is browsing objects from the set OS. There exists a ranking (or permutation) function $\sigma$, s.t. $\sigma\left(o_{1}\right) \geq \sigma\left(o_{2}\right) \geq \cdots \geq \sigma\left(o_{N}\right)$ in accordance with the preferences of the user. We explore the practical feasibility of designing an algorithm $A$, such that $A(S, O S) \sim \sigma$. Specifically, in this work, we consider $N$ to be 3 , and present the ranking algorithm and its performance on ranking 3 consumer objects.

\subsection{Related Work}

In a consumer shopping task, [5] explored the ERP measures and the role of math anxiety in consumers for discounted and promotional products. The correlation of different EEG frequency bands with the subject's internal decision of like or dislike towards the product has been shown in [6]. They concluded that theta band activity near frontal, parietal and occipital lobes are reflective of human preferences. [7] establishes the feasibility of detecting subjecting preferences through N200 signals, LPPs and Positive Slow Waves (PSWs). Moreover, the authors found that subsequent buying decisions also modulated the LPPs. [8] reported an average accuracy of $60 \%$ when predicting the preferred product from a pair of products using N200 and theta wave features. [9] classified 30 pairs of shoes successfully in two classes (buy and no-buy) for 40 participants. [10] developed a predictive modeling framework to understand consumer choices towards e-commerce products from 14 categories ( 3 products each). An accuracy of $70.33 \%$ was achieved for the consumer choice classification task using S-Golay filtering, Discrete Wavelet Transform (DWT) coupled with Hidden-Markov Models (HMMs).

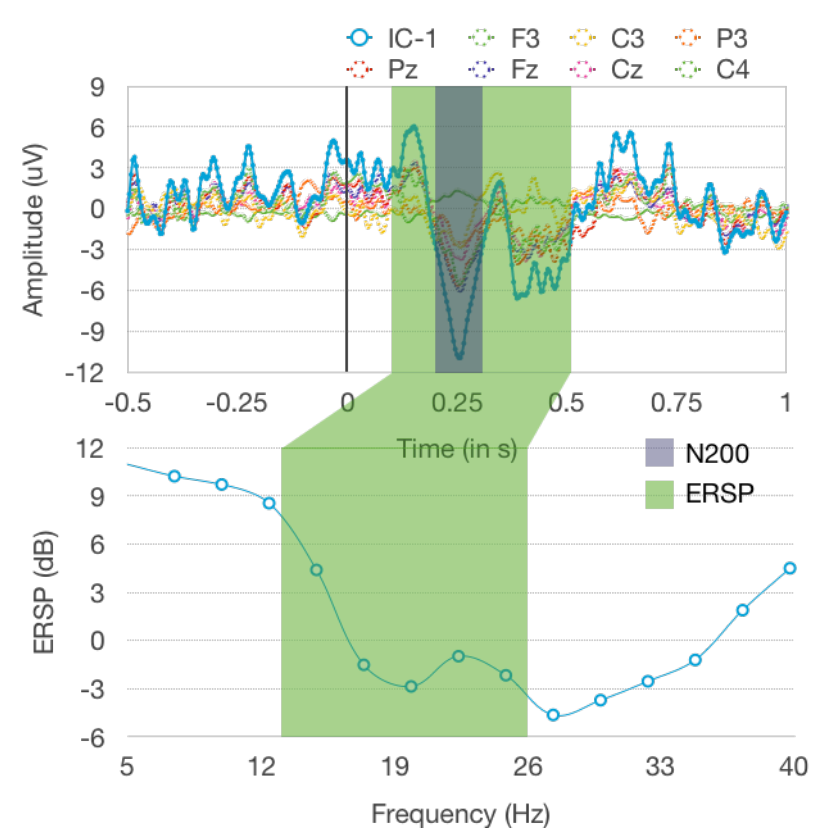

Figure 3: EEG waveform and features

\section{FEASIBILITY OF OBJECT RANKING USING EEG}

\subsection{Dataset}

We rely on the dataset obtained through the experiments in [8] to perform our analysis. In [8], the experimental design involves a pairwise classification task where 14 subjects were shown 10 unique consumer products ${ }^{2}$ and their neural activity was recorded simultaneously. Later in the experiment, the subjects were shown 2 random products side-by-side (out of 10) and were asked to choose and label the more preferable product. The first part of the experiment was repeated 50 times for each product (per subject) and provides the raw neural signals. The latter part of the experiment included 45 unique product-pairs, and each product-pair was repeated 6 times to tackle the stochasticity in consumer preferences, which serves as ground truth labels for ranking and preference scores of the products (out of 54).

\subsection{Feature Design}

The source of the neural signals associated with the user preferences is known to be located in the fronto-central region. Hence, we performed the channel selection with F3, C3, P3, Pz, Fz, Cz, C4 electrodes according to the 10-20 electrode system. A cleaner ERP signal is obtained by decomposing the channel data in the independent components and obtaining the top component through the FastICA algorithm (Fig. 3). For simplicity, we will use the term waveform to mention IC-1 component of the EEG signal. From this waveform, we extract three features to capture the user preference information for predictive analysis:

${ }^{2}$ In the conducted experiment, the objects were consumer products. In the rest of the paper, we use the terms products and objects interchangably 
- N200 mean: The mean amplitude of the waveform is computed in the time interval of $200 \mathrm{~ms}$ to $300 \mathrm{~ms}$ (fig. 3).

- N200 minima: We also consider the minimum amplitude of the N200 interval as an additional feature.

- Event Related Spectral Power (ERSP): The power spectral density of the waveform is calculated in the time interval of $100 \mathrm{~ms}$ to $400 \mathrm{~ms}$ in the beta frequency range i.e. 13 to $26 \mathrm{~Hz}$ (fig. 3). This PSD is calculated relative to the pre-stimulus baseline of $500 \mathrm{~ms}$.

We compute the Pearson correlation coefficient to explore the relationship between $N 200$ and ERSP features. We obtained a correlation coefficient of 0.0025 for N200 mean and ERSP indicating that the features are uncorrelated $(\mathrm{p}$-value $=0.0237<0.05)$. During our brute force trial and error analysis, we found that the combination of these features presents the most distinctive variability in the predictive analysis. The utility of N200 mean and ERSP in beta band for predicting user preferences is also reported in [7, 8] and [11] respectively.

\subsection{Establishing Feasibility}

In this subsection, we use the aforementioned features in the EEG signals to determine pairwise preference with two objects at a time. We thus establish the feasibility of rank ordering the objects using the pairwise results.

The task of pairwise choice classification involves mapping the neural measurement orderings to the preference amongst the consumer products. Thus, each neural feature (i.e. N200 mean, minima and $E R S P$ ) is independently used to predict the more preferred product in each product-pair. Specifically, the products having higher ERSP or higher magnitude of N200 mean were found to have a lower preference, and the products with higher N200 minima had a higher preference. These comparison rules provide an accuracy of $63.38 \%$, $64.01 \%$, and $59.40 \%$ respectively for N200 mean, N200 minima, and ERSP.A voting classifier combining all three features performed with an accuracy of $66.7 \%$. These metrics were computed on all pair of products. As the difference between the preference scores between the two products compared increases, the accuracy increases as well (as can be seen in fig. 4). The maximum accuracy achieved is $82 \%$.

Once pairwise preference can be determined, a naive ranking algorithm can be designed based on the relative ordering of one of the neural features. However, combining all of the three features is not as trivial as designing the decision classifier for the pairwise classification task. In addition, a fixed-comparison rule-based ranking algorithm will be oblivious to the individual differences (e.g. users with higher ERSP variations in comparison to N200), and hence, will not be able to generalize over a large set of users. We address these issues in the next section by presenting the Cerebro solution.

\section{THE Cerebro SOLUTION}

Having established the feasibility of object ranking based on an EEG wearable, in this section, we present Cerebro, a machine learning algorithm that can learn the specific nuances of the user's waveforms for preferences, and is thus capable of ranking objects accurately.

\subsection{Ranking Algorithm}

As described in section 3, the processed data for user $u$ and product $i$, is a vector of neural features $\left(X_{u, i}\right)$ and the preference score $\left(y_{u, i}\right)$. N200 mean and N200 minima are transformed using function, $f(x)=10 \log \left(1+x^{2}\right)$, to express $N 200$ features on the same scale as of ERSP. We build on the pairwise transformation ideas of learning to rank [12], and transform our dataset for each subject as,

$$
\left\{X_{u, k}^{\prime}, y_{u, k}^{\prime}\right\}=\left\{X_{u, i}-X_{u, j}, \operatorname{sign}\left(y_{u, i}-y_{u, j}\right)\right\}, i \neq j
$$

i.e. for each product-pair, we use the relative differences in neural features, as our transformed set of features. The labels are also transformed to +1 or -1 indicating if the $i^{\text {th }}$ product was preferred more or less. This pairwise transformation enables the prediction of the relative order of products (which is critical in ranking) rather than the pointwise approach which approximates the preference scores using neural features.

Based on the results in section 3.3, the relative order of the products is assumed to be linear with the given neural features. Hence, we fit a linear regression model $^{3}$ on the transformed set of features to predict the products with higher preferences. The regression model outputs a scalar value, which if positive, can be interpreted as the $i^{t h}$ product is more preferable (or vice-a-versa, if negative). The linear model parameter $\beta$, on a conceptual level models the individual differences in terms of the importance of each feature for comprehending the user preferences. In the loss function of linear regression $L(\beta)$, we add a linear combination of L1 and L2 penalties for regularization in order to achieve a robust prediction.

$$
L(\beta)=\frac{1}{|K|} \sum_{k}^{|K|}\left\|y_{k}^{\prime}-X_{k}^{\prime} \beta\right\|^{2}+\lambda_{1}\|\beta\|_{1}+\lambda_{2}\|\beta\|^{2}
$$

L2 penalty (also known as Ridge regression) regulates the magnitude of the parameter $\beta$ to tackle the over-fitting issue. L1 penalty (also known as Lasso regression) shrinks the coefficients of less important features to zero, thus, acts as a feature selection step. The optimal $\beta^{*}$ is learned by minimizing the overall loss function eq. (2) over the training samples, $\beta^{*}=\arg \min L(\beta)$. We learn a unique and optimal $\beta_{u}^{*}$ for each subject $u$. Now, for user $u$, given the neural measure of a new product $p$ (i.e. $X_{u, p}^{\prime}$ ), the preference score can be calculated by projecting the neural feature vector onto $\beta_{u}^{*}$ i.e. $\frac{X_{u, p}^{\prime} \cdot \beta_{u}^{*}}{\left\|\beta_{u}^{*}\right\|}$. The predicted preference scores are then compared to rank order the products.

\subsection{Evaluation}

Methodology: The ElasticNet [13] model was used to combine the L1 and L2 penalties in the linear regression model. $\lambda_{1}$ and $\lambda_{2}$ were set to 0.5 . For each subject, we train the algorithm with 7 products, providing 42 training samples with pairwise transformation for the linear regression model. The algorithm was evaluated on the remaining 3 products by comparing the predicted ranking with the user-specified rankings. A total of 120 different training-testing sets are possible, hence, we present the performance metrics averaged over all the possible combinations.

\footnotetext{
${ }^{3}$ A classification model (e.g. RankSVM) is also an appropriate alternative.
} 


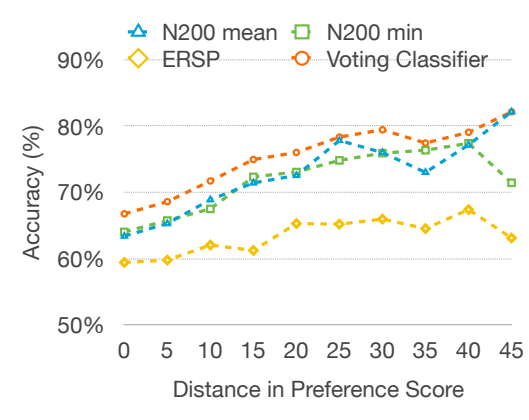

Figure 4: Accuracy for products with given distance in the preference scores

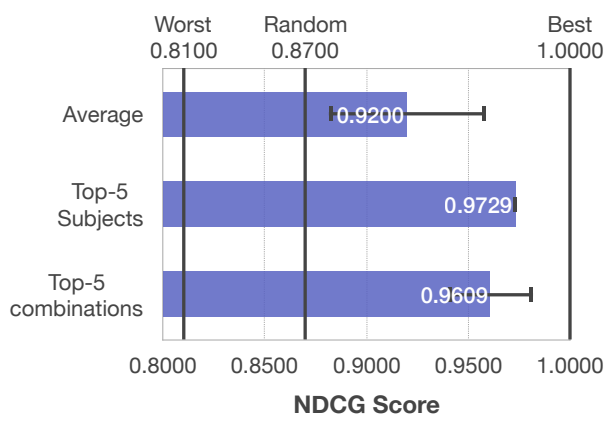

Figure 5: Ranking Score NDCG

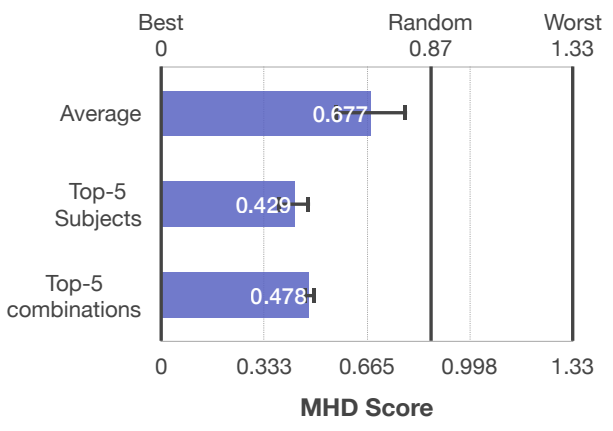

Figure 6: Ranking Score MHD
Metrics: To evaluate the performance of Cerebro, we use two metrics, namely (i) MHD Score (Mean Hamming Distance), and (ii) NDCG Score (Normalized Discounted Cumulative Gain).

- MHD Score: MHD score computes the mean hamming distance between the predicted rank and the ground truth. For e.g., if a ground truth rank of $y_{\text {rank }}=[1,2,3]$ is predicted as $\hat{y}_{\text {rank }}=[3$, $1,2]$, the MHD score would be 1.33. For 3 elements, the best, the worst, and random change MHD would be $0,1.33$ and 0.87 respectively.

- NDCG Score: It measures the ranking quality by accounting the preference of the products ranked. NDCG is computed by normalizing the DCG score (Discounted Cumulative Gain), $\sum_{i=1}^{N} \frac{r e l_{i}}{\log _{2}(i+1)}$, with ideal DCG score. Here, rel $_{i}$ represents the preference of the ${ }^{t h}$ product. An ideal DCG score would be the DCG score of products when ranked according to their preferences. For e.g., if the products with preference scores of $y_{r e l}=[30,20,10]$ are ranked as $\hat{y}_{\text {rank }}=[3,1,2]$, the DCG score would be 41.31 , with an ideal DCG of 47.61, giving NDCG as 0.867 . An NDCG score of 1.0 is ideal. For the preference scores in our dataset, a random chance NDCG is 0.87 .

Performance: Fig. 5 and 6 shows the ranking performance on NDCG and MHD scores respectively. On an average (14 subjects, 120 training combinations), the ranking algorithm performs with an NDCG score of $0.92( \pm 0.11)$ and MHD score of $0.67( \pm 0.03)$. The considerable standard deviation in the ranking performance is due to the high variability of ranking performance across subjects and training combinations. Hence, we also evaluate the performance of top-5 subjects and top- 5 training combinations. For top- 5 subjects, the performance jumps to $0.973( \pm 0.0007) \mathrm{NDCG}$, and $0.429( \pm$ 0.052) MHD. Similarly, for top-5 training set combinations, we achieve $0.961( \pm 0.02)$ and $0.477( \pm 0.02)$ respectively.

\subsection{Determination of Confidence in Ranking}

Note that Cerebro requires user-training to understand and subsequently predict user preferences. One of the key questions that arises is the following - when is the algorithm trained enough such
Table 1: Confidence in Training

\begin{tabular}{l|l|l}
\hline & Top-10 & Worst-10 \\
\hline \hline Training MHD & 0.731 & 0.199 \\
\hline Testing MHD & 0.476 & 0.876 \\
\hline
\end{tabular}

that it can start recommending objects according to the user preferences (i.e. when the neural signal based estimated preferences are actionable in real-world deployment)?. From the discussions thus far, we can observe that the performance of the ranking algorithm depends on the subjects and the set of product combinations chosen for training the algorithm. In this subsection, we explore whether it is feasible for the algorithm to self-determine if it has encountered the right set of products to be effectively trained. If such self-determination is feasible, the algorithm can begin predicting ranks for new objects only when it is sufficiently confident of its training.

Fig. 7 shows the average MHD score (over all 14 users) with respect to the mean rank of the 7 products used in the training. With a larger spread of product ranking in the training set (mean training rank close to 5.5), it performs significantly better than with training products that are heavily biased towards top (or bottom) ranks. If the top 7 products are considered for training, it performs $20.1 \%$ worse than a uniform spread of training products (with a mean ranking of 5).

For practical self-determination of its confidence, the confidence measure should be solely based on the set of training products encountered thus far. In our dataset, we find that the combinations performing comparatively poor (in terms of training score of MHD or NDCG), tend to perform highly accurate on the testing data. A possible reason for this trend could be that the algorithm is exposed to the data with more variations, hence the training fit is reasonable (no overfitting), but more generalized to the unseen data. Table 1 presents the MHD score of training combinations which has top-10 and worst-10 training accuracy. These results, while preliminary, shed light on an approach to predict confidence 


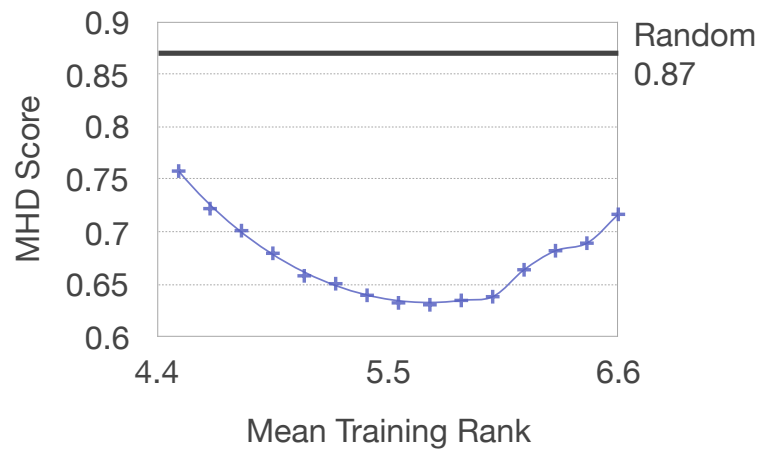

Figure 7: MHD Score on different training combinations

in performance for unseen products. When the training accuracy is tracked over time (with more number of products), the training and testing accuracy converge, indicating the confidence. Our analytical approach is limited because of the small size (10 products) of the dataset. Another ideal approach to obtain confidence is through cross-validation [14]. The verification of this methodology is left for future work.

\subsection{System Architecture}

In this subsection, we describe the system design allowing Cerebro to understand user preferences. There are three main components of the system architecture, namely, (i) wearable device, (ii) mobile software, and (iii) cloud server. The wearable device detects EEG signals and ships the digitized signals to the user's mobile device through a wireless link. The mobile software running on the user's mobile device processes the raw signals and extract neural features related to the user preferences as described in section 3.1 and 3.2. The computed features are sent to the cloud server which executes the Cerebro algorithm to understand the user preferences and thus, ranks the objects. Finally, the analytical summary of the user preferences and ranking is sent to the concerned application server (e.g. Amazon personalization engine).

\section{CONCLUSIONS}

This paper considers the potential of tracking neurobiological changes through wearable EEG headsets to understand user preferences. We study the detection and interpretation of user brainwaves to rank a given set of objects based on user preferences. We present Cerebro, a machine learning algorithm to enable objects ranking merely through the neural data based on user preferences. The performance of Cerebro is attractive, with an NDCG score of 0.92 .

In terms of future work, we intend to extend this work in two main directions - (i) validating and evaluating Cerebro over a large corpus of user preference EEG data, and (ii) observing the performance of the algorithm in real-life conditions when the users are actually browsing products on their mobile devices.

Acknowledgements: We thank the authors of [8] for providing us access to the EEG dataset and their guidance for maneuvering through the dataset. This work was supported in part by the
National Science Foundation under grants 1701115, 1813242, and 1837369, and the Wayne J. Holman Chair.

\section{REFERENCES}

[1] Danny Oude Bos et al. Eeg-based emotion recognition. The Influence of Visual and Auditory Stimuli, 56(3):1-17, 2006.

[2] Ning-Han Liu, Cheng-Yu Chiang, and Hsuan-Chin Chu. Recognizing the degree of human attention using eeg signals from mobile sensors. Sensors, 13(8):1027310286, 2013.

[3] Niko A Busch, Julien Dubois, and Rufin VanRullen. The phase of ongoing eeg oscillations predicts visual perception. Fournal of Neuroscience, 29(24):7869-7876, 2009.

[4] Meng-Hsien Lin, Samantha NN Cross, William J Jones, and Terry L Childers. Applying eeg in consumer neuroscience. European fournal of Marketing, 52(1/2):6691, 2018.

[5] William J Jones, Terry L Childers, and Yang Jiang. The shopping brain: Math anxiety modulates brain responses to buying decisions. Biological Psychology, 89(1):201-213, 2012.

[6] Rami N. Khushaba, Luke Greenacre, Sarath Kodagoda, Jordan Louviere, Sandra Burke, and Gamini Dissanayake. Choice modeling and the brain: A study on the electroencephalogram (eeg) of preferences. Expert Systems with Applications, 39(16):12378 - 12388, 2012.

[7] Nobuhiko Goto, Faisal Mushtaq, Dexter Shee, Xue Li Lim, Matin Mortazavi, Motoki Watabe, and Alexandre Schaefer. Neural signals of selective attention are modulated by subjective preferences and buying decisions in a virtual shopping task. Biological Psychology, 128:11 - 20, 2017.

[8] Ariel Telpaz, Ryan Webb, and Dino J Levy. Using eeg to predict consumers' future choices. Fournal of Marketing Research, 52(4):511-529, 2015.

[9] Davide Baldo, Hirak Parikh, Yvonne Piu, and Kai-Markus MÃijller. Brain waves predict success of new fashion products: A practical application for the footwear retailing industry. Journal of Creating Value, 1(1):61-71, 2015.

[10] Mahendra Yadava, Pradeep Kumar, Rajkumar Saini, Partha Pratim Roy, and Debi Prosad Dogra. Analysis of eeg signals and its application to neuromarketing. Multimedia Tools and Applications, 76(18):19087-19111, Sep 2017.

[11] Jonghyeok Park, Hackjin Kim, Jeong-Woo Sohn, Jong-ryul Choi, and Sung-Phil $\mathrm{Kim}$. Eeg beta oscillations in the temporoparietal area related to the accuracy in estimating others' preference. Frontiers in Human Neuroscience, 12:43, 2018.

[12] Thorsten Joachims. Optimizing search engines using clickthrough data. In Proceedings of the eighth ACM SIGKDD international conference on Knowledge discovery and data mining, pages 133-142. ACM, 2002.

[13] Hui Zou and Trevor Hastie. Regularization and variable selection via the elastic net. Journal of the royal statistical society: series B (statistical methodology), 67(2):301-320, 2005.

[14] Ron Kohavi et al. A study of cross-validation and bootstrap for accuracy estimation and model selection. In Ijcai, volume 14, pages 1137-1145. Montreal, Canada, 1995. 